\title{
EDITORIAL
}

\section{THAT TOUCH OF STYLE}

It sometimes seems that all the emphasis is on streamlining the pipeline to speed up the

discovery and development process. Is that other essential element being ignored?

If you have ever been the fortunate recipient of any management training, it is a near certainty that you will have been cajoled into some form of group activity. Perhaps it was passing a balloon round the group without using your hands, or maybe struggling round the parking lot together on large planks, but whatever form the embarrassment took, the purpose was undoubtedly to demonstrate how much more efficiently a team can complete a task than an individual. In these exercises, 'efficiency' seems to be equated with speed. No points are given for 'style'. Translating this to the world of drug discovery and development, the current emphasis on speeding up the pipeline seems to neglect the essential contribution of scientific style.

Speedier drug production is certainly needed. The greatest internal industry drivers for this are expense, and the ever diminishing period of market exclusivity that companies can expect from their new products. When Inderal (propranolol) was launched in 1968, it had the market to itself for ten years before the next beta blocker, Lopressor, came along. Prozac had four years to enjoy the market following its launch in 1988, before the advent of the next SSRI, Zoloft. But when the COX2 inhibitor Celebrex was launched in 1999, it was being jostled from its podium by its 'fast follower' Vioxx within four months. Such competition in the market makes a strong case for moving things along faster. Pressure for speed comes from outside the industry too, with rising patient expectations being reflected in the new power wielded by patient advocacy groups.

In response, cycle times in the drug business are diminishing. Most effort has been directed towards pushing down the length of the most expensive part of the process - the development phase. Average times for the completion of clinical trials are dropping steadily, although the long and costly enrolment phases of trials are proving resistant to further reduction. Initiatives abound to decrease development times further, among the most interesting being the New Safe Medicines Faster programme from the European Federation for Pharmaceutical Sciences, which aims to bring academia and industry together to reinvigorate the climate for drug development in Europe. But the most dramatic time savings over the past decade or so have in fact been made by the regulatory authorities.

Putting aside last year's high-profile and recordbreaking approval of Novartis' kinase inhibitor Gleevec in under three months, the average time for approval of drugs accorded priority review by the FDA - those drugs that offer "significant improvement compared to marketed products" - was just six months. Approval times in general have approximately halved over the past decade. But does increased speed lead to 'more efficient' production of new drugs?

Last year saw a record low in the number of new drug approvals, at least in most people's memory. Just 24 of the drugs approved by the FDA were new molecular entities (NMEs), rather than reformulations or the use of old drugs for new indications. Thirty-one NMEs were launched worldwide, but only a handful of these fulfilled the clinical criteria of 'priority' drugs. Although a snapshot of a single year tells only a partial story, the increased speed of the drug discovery and development pipeline does not, as yet, seem to have contributed to its productivity. Everywhere you look, you see exciting new therapies being investigated, so where do all the drugs go? If you take a glance at the Obesity Market Overview on pages 257-258 of this issue, you will see a list of 13 drugs in clinical development, as well as 7 promising looking ideas at the preclinical stage. One wonders how many of them will make it to market?

Of course, we all know that rapidity is only part of the solution. But attend any briefing on 'the future of drug discovery and development', and you'll hear a lot about 'tackling the bottlenecks' and 'benchmarking' the process. Important, no doubt, but once all the elements are streamlined to the last degree, we'll be left with the obvious fact that it is the quality of the underlying science that makes the difference between success and failure. So how do you 'benchmark' the science that underpins the process? That might be said to be our business at Nature Reviews Drug Discovery - trying always to select the most promising topics and assess them in the most rigorous way. 